(Received 4 July, 2005; Revision Accepted 25 April. 2006)

\title{
ABSTRACT
}

The generalized model of the blood glucose/insulin concentration in an insulin-dependent diabetic patient where the patient has variable pancreatic response io glucose level was considered. It was shown that greater response of the pancreas to glucose concentration leads to quicker clearance of excess glucose in the blood. Also, the gieatest quantity of glucose to be found at any time when the response is high is less when compared with that of lower response of the pancreas to the glucose level. Another disturbing result is the fact that if the pancreatic response to glurose level (and thus level of insulin release) is non-steady, we then have dual or multiple peak of insulin level, winich present variable insu! in production for those with higher pancreatic response. This results to problem as regards the conversion mode of the glucose to glycogen. The consequence of this is that the patient might be exposed to such situations like low blood glucose level and might then suffer hypoglycemia if proper diagnosis was not made to determine the exact diabetic level or severity.

The conclusion derivable here therefore is that appropriate control of the blood glucose level will not be possible by insulin infusion since the correct blood insulin level

can not be accurately estimated at any time.

KEYWORDS: Mathematical model, Blood, Glucose, Insulin, Pancreas, Diahetic Patient. INTRODUCTION

Diabetes mellitus is a disease characterized by chronic high blood plasma glucose concentration and other disturbances of carbohydrate and lipid metabolism in man. Depending on the level of severity of this disease, the plasma glucose concentration can be as high as $200 \mathrm{mg} / 100 \mathrm{cc}$ or even more as against the normal plasma g ucose level of between $70-115 \mathrm{mg} / 100 \mathrm{cc}$ in an overnight fasting. (Ellenberg and Rifkin, 1983). This high plasma glucose concentration usually results to micro-vascular complications of which the most importantly affected parts of the numan system are the eyes and the kidney. (Czech and Massagnes, 1982 , Vallence-Owen, 1975 ).

There are various causes of diabetes mellitus though they are put into two broad forms: insulin -dependent and the non-insulin -dependent. Of particular interest in this work is the insulin-dependent form. The insulindependent diabetes mellitus is a type of disease caused by low insulin concentration in the blood plasma of which the simplest approach to treatment is the insulin infusion or administration. Insulin is a hormone secreted by the islet of langerhans of the pancreas. It has wide spectrum of activities among, which is the enhancement of the cell conversion of the excess glucose in the plasma to glycogen, which is the storable form of glucose in the body organs and cells (Mbah, 1998). It is this particular attribute of the insulin that is usually lacking in an insulin-dependent diabetic patient. In norma! persons, carbohydrate taken as food is digested in the alimentary canal from where the glucose and other monosacharides are absorbed into the blood. From the blood, they diffuse into the extra-cellular fluids. By facilitatedmediated transport process, this glucose is taken to the cell plasma membrane by the carrier proteins called glucose transporters (Simpson and Cushman, 1985): About six of such glucose transporters have been identified (Beil et al . 1989). The cell membrane structure shows that the outer surface of the plasma membrane is lined with evenly distribution protein receptors for hormones and other chemical messengeis (Czech and Massagnes, 1982, Gavin et al

1974, Kosmokos and Roth, 1980, Lorinth and Smith, 1983). This makes the plasma membrane a signal -receiving device for the detection of chemical signals that regulate the cell activities. It is at this membrane that the insulin performs its function by binding with these receptor proteins either in phosphorylated or unphosphorylated form.s. It is this autophosphorylation action of the insulin that causes the aggregation of the protein receptors at the plasma membrane (Quon and Campfield, 1985). This reduces the inhibitory action of these proteins to the entry of glucose into the cells. Although the exact mechanism of glucose entry into the cells is not clearly known, it is thought that the giucose, through the facilitated-mediated transport, gets to the plasma membrane where they bind with the appropriate carrier proteins leading to conformal changes in the carrier molecules that will now allow the passage of the glucose. Though this is what happens in the blood/ cells of a normal person, it is not exactly the same in an insulin-dependent diabetic patient. In this case, there is usually low concentration of insulin in the plasma. This affects the proper functioning of the insulin with particular reference to the plasma glucose regulation, (Bute, 1982). Usually the low plasma insulin concentration is occasioned by reduced response of the pancreatic cells to produce/release insulin when stimulated by high plasma glucose concentration. This action leads to reduced interaction of the plasma glucose and insulin and thus the high plasma concentration of the glucose. 
A mathematical model showing the yariation in the level of the glucose or insulin in the blood of an insulindependent diabetic patient was presented (Mbah, 1998 ).

In summarized form we state the factors that affect the levels of glucose and insulin in the blood at all times. We have that for the blood glucose level, the factors that determines it are:

the quantity of food taken,

$\therefore \quad$ glucose/insulin interactian (in other words the glucose conversion to glycogen

due to peripheral cell response),

the amount of this glucose being converted to energy and finally

the release of the stored glycogen by the liver for a long period of no food. intake.

For the insulin level, we see that the factors are the pancreatic cell response to the level of glucose in the blood, the insulin usage and the insulin infusion because the patient is diabetic.

Detailed study on the derivation of the mathematical models can be obtained in Mbah (1998), Mbah (2001). The resulting mathematical models are:

$$
\begin{aligned}
& \frac{d x}{d t} \doteq \dot{a}_{1}=(l)-a_{2} x y-a_{3} x+a x\left(x_{0}-x\right)----------(1) \\
& \frac{d y}{d t}=b_{1} x-b_{2} y+b_{3} w(t)----------------(2) \\
& \text { where } \mathrm{x}=\text { glucose level } \\
& y=\text { insulin level } \\
& z(t)=Q e^{-k\left(t-t_{1 .}\right)} \quad \text { and } \\
& W(t)=\left\{\begin{array}{cl}
\frac{w_{0}\left(t-t_{0}\right)}{i-t_{0}}, & t_{0}<t \leq i \\
\frac{-w_{0}\left(t-\left(2 i-t_{0}\right)\right)}{i-t_{0}}, & i \leq t \leq 2 \bar{i}-t_{0} \\
0 & \text { elsewhere. }
\end{array}=\gamma \mathrm{t}+\delta\right.
\end{aligned}
$$

Thie constants $a_{1}, a_{2}, a_{3}, a_{4}$ and $b_{1}, b_{2}, b_{3}, \delta_{1} \gamma$ are associated with the behaviour of the glucose and insulin to the system, of the individual concerned respectively.

Equation (1) as presented is a general case but we shall assume that the patient is not starving so that $a_{4}\left(x_{0}\right.$ $-x)$ will be neglected. Thus our equations of interest will be

$$
\begin{aligned}
& \frac{d x}{d t}=a_{1} z(t)-a_{2} x y-a_{1} x-\ldots-\ldots-\ldots-\cdots-\cdots(3) \\
& \frac{d y}{d t}=b_{1} x-b_{2} y+b_{3} w(1)
\end{aligned}
$$

Now a look at equation (3) shows that it is non-linear. However, since_the patient is not starving meaning that there is always enough glucose in the blood to be converted to glycogen in the presence of insulin, we may linearize the term ry to $\bar{x} y$ where $\bar{x}$ is now a constant. With this, equation (3) now becomes a linear differential equation and given as:

$$
\frac{d x}{d t}=a_{1} z(t)-a_{2} \bar{x} y-a_{3} x
$$

\section{Method of solution}

The equations (4) and (5) are first order differential equations and are to be solved simultaneously. Substituting as given above, we have

$$
\begin{aligned}
& \frac{d x}{d t}=a_{1} Q^{-k\left(t-l_{c}\right)}-a_{2} \bar{x} y-a_{3} x \\
& \frac{d y}{d t}=b_{1} x-b_{2} y+b_{3}(x+\delta) .
\end{aligned}
$$


In equations (6) and (7), $a_{2}$ and $b_{1}$ measures the glucose and insulin interaction and the pancreatic response to the glucose stimulation respectively. Hence, the severity of the patient's diabetes depends principally on how smal the value of $b_{1}$ is and also on the value of $a_{2}$. These two equations are solved simultaneously by finding thei: complementary and particular solutions which when added up give their solutions. Suppose the complementan solutions $(x, y)=\left(x_{c}, y_{c}\right)$ to the homogenous equations.

$$
\begin{aligned}
& \frac{d x}{d t}=-a_{2} \bar{x} y-a_{3} x \\
& \frac{d y}{d t}=b_{1} x-b_{2} y
\end{aligned}
$$

is given by

$$
\left(\begin{array}{l}
x_{c} \\
y_{c}
\end{array}\right)=\left(\begin{array}{l}
\xi_{1} \\
\eta_{2}
\end{array}\right) e^{\lambda_{1} \prime}+\left(\begin{array}{l}
\xi_{2} \\
\eta_{2}
\end{array}\right) e^{\lambda_{2} \prime}
$$

From equations (8) and (9) we have the matrix of coefficients as

$$
T=\left(\begin{array}{cc}
-a_{3} & -a_{2} \bar{x} \\
b_{1} & -b_{2}
\end{array}\right)
$$

The eigen-values $\lambda_{1}$ and $\lambda_{2}$ of matrix $T$ are got by sotving for $\lambda$ in the determinantal equation

Hence, we have

$$
\left|\begin{array}{cc}
-a_{3}-\lambda & -a_{2} \bar{x} \\
b_{1} & -b_{2}-\lambda
\end{array}\right|=0
$$

$$
\lambda_{1,2}=\frac{1}{2}\left[-\left(a_{3}+b_{2}\right) \pm\left\{\left(a_{3}+b_{2}\right)^{2}-4\left(a_{3} b_{2}+a_{2} b_{1} \bar{x}\right)\right\}^{\frac{1}{2}}\right]
$$

We must find the particular solutions for the equations in terms of, $z(t)$ and $\omega(t)$ respectively. Let these particuli solutions be denoted by

$$
\left(\begin{array}{l}
x_{z} \\
y_{z}
\end{array}\right) \text { and }\left(\begin{array}{l}
x_{w} \\
y_{w}
\end{array}\right)
$$

Thus, for $z(t)$, we solve to get the particular solution as:

$$
\left(\begin{array}{c}
x_{z} \\
y_{z}
\end{array}\right)=\frac{1}{D}\left(\begin{array}{c}
a_{1} Q\left(b_{2}-k\right) \\
a_{1} b_{1} Q
\end{array}\right) e^{-k\left(t-t_{0}\right)}
$$

For.w(t), we also solve to get the particular solution as

$$
\therefore\left(\begin{array}{c}
x_{w} \\
y_{w}
\end{array}\right)=\frac{1}{m}\left(\begin{array}{c}
-a_{2} \bar{x} \\
a_{3}
\end{array}\right) \gamma t+\left(\begin{array}{r}
\frac{a_{2} \bar{x} \gamma\left(b_{2} a_{3}+a_{3}^{2}\right)}{a_{3} m^{2}}-\frac{a_{2} \bar{x} \delta}{m} \\
\frac{\delta a_{3}}{\dot{m}}-\frac{\gamma}{m}\left(a_{3}^{2}-a_{2} b_{1} \bar{x}\right)
\end{array}\right)
$$


In general therefore, the solution to the equation is

When eqfations (10), (13) and (14) are substituted into equation (15) we have

$$
\begin{aligned}
x & =\varepsilon_{1} e^{\lambda_{1} t}+\varepsilon_{2} e^{\lambda_{2} t}+a_{1} Q \frac{\left(b_{2}-k\right) e^{-k\left(t-t_{0}\right)}}{z}-b_{3} \frac{\left(a_{2} \bar{x} \gamma t\right)}{m} \\
& +\frac{b_{3} a_{2} \bar{x} \gamma\left(b_{2} a_{3}+a_{3}^{2}\right)}{a_{3} m^{2}}-\frac{b_{3} a_{2} \bar{x} \delta}{m} \\
y & =\frac{\left(\lambda_{1}+a_{3}\right) \varepsilon_{1} e^{\lambda_{1} t}}{a_{2} \bar{x}}-\frac{\left(\lambda_{2}+a_{3}\right) \varepsilon_{2} e^{\lambda_{2} t}}{a_{2} \bar{x}}+\frac{a_{1} b_{1} Q e^{-k\left(1-t_{0}\right)}}{z} \\
& +\frac{b_{3} a_{3} \gamma t}{m}+\frac{b_{3} \delta a_{3}}{m}-\frac{b_{3} \gamma\left(a_{3}^{2}-a_{2} b_{9} \bar{x}\right)}{m^{2}}
\end{aligned}
$$

where $\mathrm{z}=\mathrm{k}^{2}-\left(b_{2}+a_{3}\right) k+a_{3} \dot{b}_{3}+a_{2} b_{1} \bar{x}$

$$
m=b_{2} a_{3}+a_{2} b_{1} \bar{x}
$$$$
\lambda_{1}=0.5\left[-\left(a_{3}+b_{2}\right)+\left(\left(a_{3}+b_{2}\right)^{2}-4\left(a_{3} b_{2}+a_{2} b_{1} \bar{x}\right)\right\}^{\frac{1}{2}}\right]
$$$$
\lambda_{2}=0.5\left[-\left(a_{3}+b_{2}\right)-\left\{\left(a_{3}+b_{2}\right)^{2}-4\left(a_{3} b_{2}+a_{2} b_{1} \bar{x}\right)\right\}^{\frac{1}{2}}\right]
$$

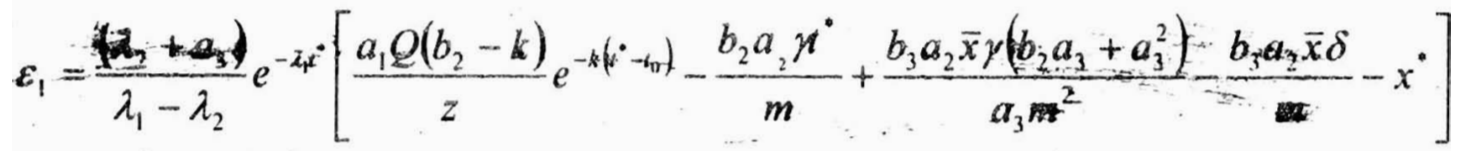

$$
\begin{aligned}
& +\frac{\left(a_{2} \bar{x} e^{-\lambda_{2} i^{*}}\right.}{\lambda_{1}-\lambda_{2}}\left[\frac{a_{1} b_{1} Q e^{-k\left(i^{*}-t_{0}\right)}}{2}+\frac{b_{3} a_{3} x^{*}}{m}+\frac{a_{3} b_{3} \delta}{m}-\frac{b_{3} \gamma\left(a_{3}^{2}-a_{2} b_{i} \bar{x}\right)}{m^{2}}-Y^{*}\right]
\end{aligned}
$$

$$
\begin{aligned}
\varepsilon_{2}= & \frac{\lambda_{2}+a_{3}}{\lambda_{2}-\lambda_{1}} e^{-\lambda_{1} i^{*}}\left[\frac{a_{1} Q\left(b_{2}-k\right)}{z} e^{-k\left(i^{*}-t_{0}\right)}-\frac{b_{3} a_{2} \bar{x} \gamma t^{*}}{m}+\frac{b_{3} a_{2} \bar{x} \gamma\left(b_{2} a_{3}+a_{3}^{2}\right)}{a_{3} m^{2}}-\frac{b_{3} a_{2} \bar{x} \delta}{m}-x^{*}\right] \\
& +\frac{a_{2} \bar{x} e^{-\lambda_{2} i^{*}}}{\lambda_{2}-\lambda_{1}}\left[\frac{a_{1} b_{1} Q e^{-k\left(i^{*}-t_{10}\right)}}{z}+\frac{b_{3} a_{3} \gamma t^{*}}{m}+\frac{a_{3} b_{3} \delta}{m}-\frac{b_{3} \gamma\left(a_{3}^{2}-a_{2} b_{1} \bar{x}\right)}{m^{2}}-Y^{*}\right]
\end{aligned}
$$

Here, $t$ represents the time of interest and can assume various values as we may desire such as $t^{\circ}=t_{0}$ which in this case will mean wishing to know what happens to the glucose and insulim levels at the beginning of the experiment denoted by $t_{0}$. The essence of the time $t$ other than $t_{0}$ is that $\varepsilon_{1}$ and $\varepsilon_{2}$ are to be determined at times other than $t_{0}$ most especially as we now have insulin infusion to determine the plasma glucose and insulin levels. Recall from the expression for the $\omega(t)$ that insulin has a $t_{1 / 2}$ value that presents different glucose response to the tevels of the insulin at such a time. The $x^{*}$ and $y^{*}$ stands for the amount of glucose and insulin in the plasma at such a time $t^{*}$ respectively. The change in $x^{*}$ and $y^{*}$ have the same importance as stated in the case of $t^{*}$.

\section{ANALYSIS}

In the analysis here, we wish to see what happens for a case where the pancreatic insulin release varies. By this, it can be likened to looking at the blood glucose/insulin levels of diabetic patients of different levels of severity that are being fed similarly with similar insulin infusion. It can also be likened to a diabetic patient whose insulin release is not steady but varies at different times within the same period of feeding. Theoretically, we know that if two patients of different levels of severity are fed with the same quantity of food (same glucose worth) and treated with equal quantity of insulin; they will present reactions to this and will have different levels of glucose/insulin, differing clearance time of th, a glucose etc. 
Using the model and the resulting solution, we tried to replicate this result. In figure (1), we considered a case where $z_{2}, b_{3}, a_{2}$ and $a_{3}$ are constant while $b_{1}$ varies. The values of $a_{2}, a_{3}, b_{2}, b_{3}$ are given: From the graph, we can see that or $b_{1}=0.05,0.1,0.2$ and 0.25 , we can see that both the glucose levels and the clearance time varied. For $b_{1}=0.25$,

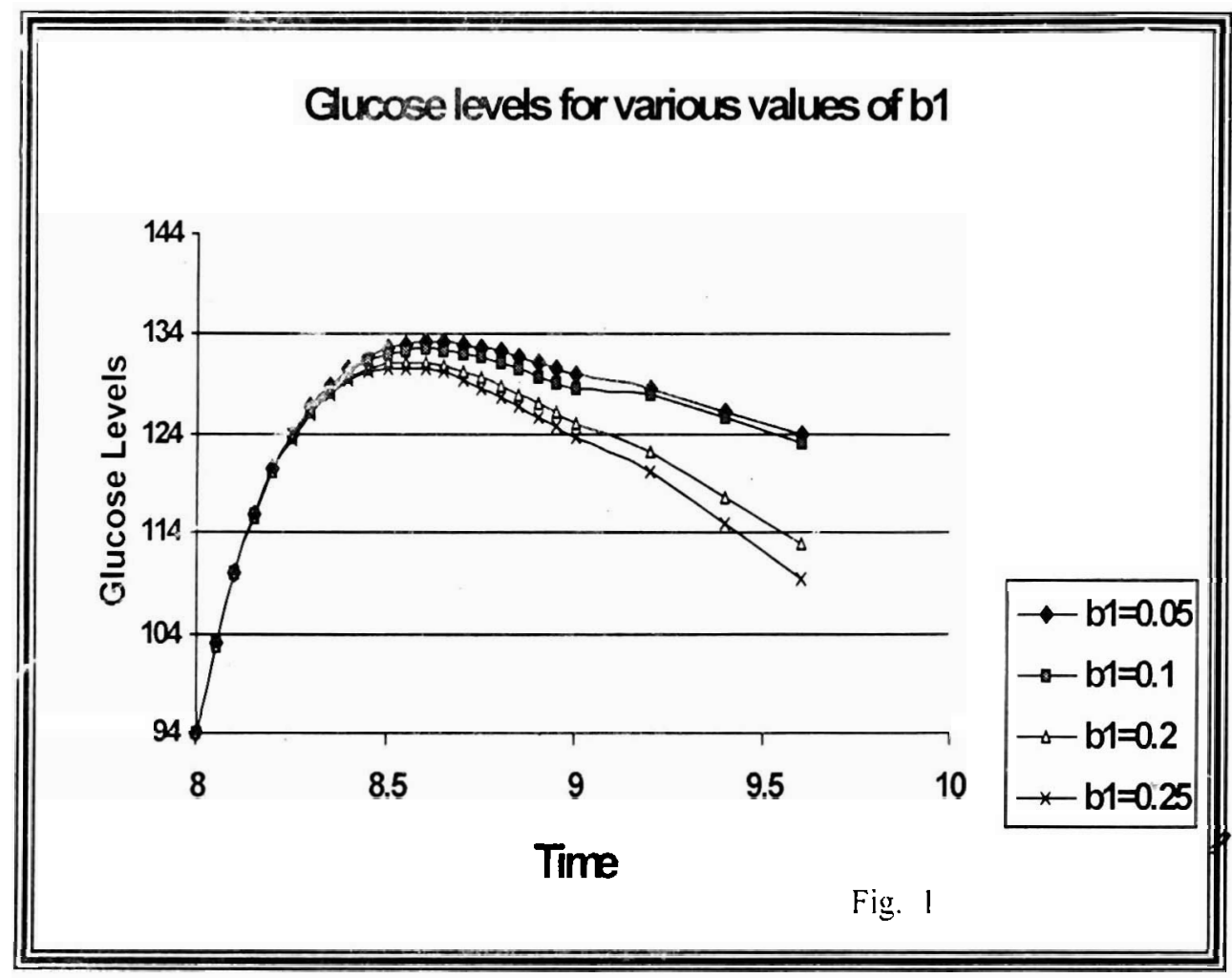

meaning greater pancreatic response to presence of glucose, the glucose level in the blood is lower than the case where $b_{1}=0.05$. This is because greater response of the pancreas means more retease of insulin to the blood and therefore more interaction of the glucose with the insulin. This greater interaction means greater reduction in the quantity of glucose in the blood by conversion into glycogen. The converse is the case in the case of $b_{1}=0.05$. We can equally see that the clearance time of the entire glucose from the blood is larger in value for the case of $b_{1}=0.05$ than in $b_{1}=0.25$ because less quantity of insulin is in the blood and thus less interaction with glucose which subsequently leads to longer time of clearance.

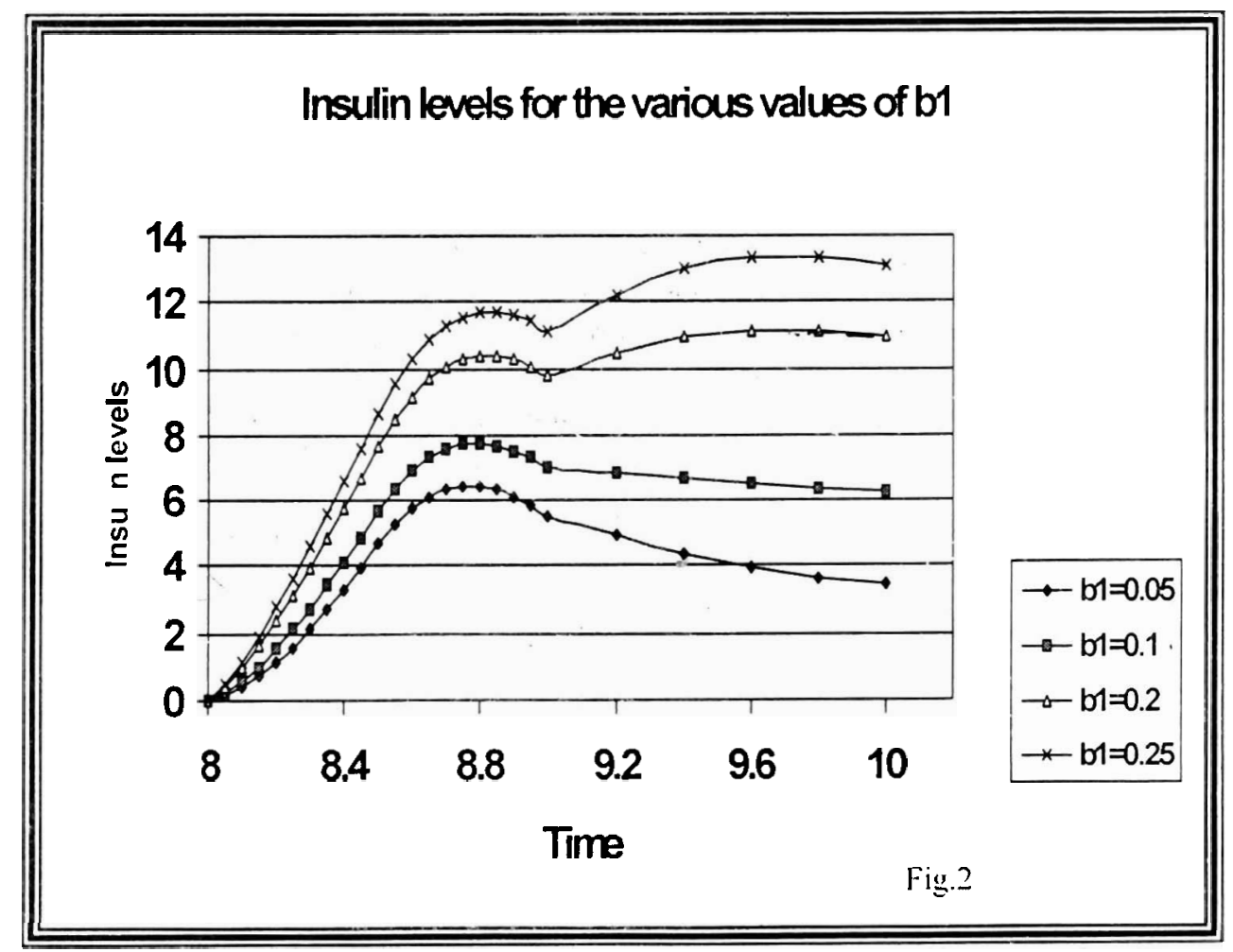


In fig. (2), we show the corresponding insulin level in the blood for the value of $b_{1}$ and $a_{2}, a_{2}, b_{2}, b_{3}$ as used above. We can see from this graph that for $b_{1}>0.1$, the resulting curves became crooked. What this means is that at this level of diabetes, the pancreas has the capacity to produce insulin and this capacity has to be respected in trying to treat such diabetic patient by insulin infusion. Because of the infused insulin the pancreas insulin release is inhibited until this infused one is exhausted and the pancreas will then start to release insulin because of the demand for more insulin due to the level of excess glucose in the blood that is not yet converted.

The biological implication of varied pancreatic response to glucose level in the blood is that we will not be sure of the state of health of the patient. If enough quantity of food is not taken by such a patient and insulin is infused such a patient might suffer hypoglycemia as the subsequent released insulin will reduce the blood glucose level to such a low level. Therefore, we may conclude that for such a patient, it will be very difficult to control the blood glucose level since the insulin release and thus its level in the blood cannot be correctly predicted. Even the quantity of the insulin to be infused cannot also be correctly measured. Pancreatic varied insulin release should therefore be considered and handled with all sense of seriousness wherever and whenever it is noticed in any patient

\section{REFERENCES}

BELL, G. I., Buse, J. B., Fukumotc, H. and Seino, S., 1989. Glucose Transporter, Molecular Structure and function. In Genes and Gene products in the Development of Diabetes Mellitus. Basic and Clinical Aspects. Elsevier, Amsterdam, pp.287 - 298.

Bute, J. C., 1982. The insulin receptor - single function and dual effect, J. Theor. Biol. 155: $427-436$.

Czech, M. P. and Massagne, K., 1982. Sub-unit structure and dynamics of the insulin receptor, Fed. Proc. Fed. Am. Soc. Expl. Biol. 41: $2719-2723$.

Elenberg, M. and Rifkin, H., 1983. (Ed.) Diabetes Mellitus, Theory and Practice. Third Edition Vol 1 \& 2. Med Examination Pub. Company Inc.

Gavin, J. R, Roth, J., Neville, D. M., De Meyts, P. and Buel, D. N., 1974. Insulin-dependent regulation of insulin receptor concentration. A direct demonstration of cell culture, Proc. Nat. Acad. Sci. USA 71: $84-88$.

Kosmokos, F. C. and Roth, J., 1980. Insulin induced was of the insulin receptors in $1 \mathrm{M}-9$. Lymphocytes: A biological Process mediated through the insulin receptors, J. Biol. Chem. 255: $9560-9869$.

Lonnrith, P. and Smith, U., 1983. B - Adrenergic dependent down regulation of insulin binding in Rat Adipocytes, Bio - Chem. Bio - Phys. Res. Commun. 112: 972 - 979.

Mbah, G .C. E, 1998. Mathematical Modelling and Control of blood glucose/Insulin Concentrations in an insulindependent Diabetic Subject. Ph.D Thesis, University of Nigeria, Nsukka.

Mbah, G .C. E., 2001. An Analytical method of solution to the generalized mathematical model used for the şludy of Insulin- independent diabetics Mellitus, J. Nig: Math Soc., 20: 65 - 76.

Quon, M .J. and Campfield, L. A., 1991. A Mathematical Model and Computer Simulation Study of Insulin Sensitive glucose transporter regulation, J. Theor: Biol, 150: $59-72$.

Simpson, I. A. and Cushman, S.W.., 1985. Hexose transporter regulation by insulin in the isolated rat adipose cell. In: M. P. (zed (Ed.), The Molecular basis of insulin Action. Pleasure, New York, pp. $399-423$.

Vallence, J., 1975. Owen (Ed) Diabetes: Its Physiology and Biochemical basis. Pub. MTP Press Ltd. U.K. 